INDO GLOBAL JOURNAL OF

PHARMACEUTICAL SCIENCES

ISSN 2249- 1023

\title{
Extracellular Polysaccharide Isolated from Dunaliella salina having Immunomodulatory and Cytotoxic Activity
}

\author{
Mehendi Goyal, Manoj Baranwal * \\ Department of Biotechnology, Thapar University, Patiala, Punjab, India \\ Address for Correspondance: Manoj Baranwal, manoj.baranwal@thapar.edu
}

\section{Keywords}

Dunaliella salina; Immunomodulatory

; Cytotoxicity;

Extracellular

Polysaccharide.

\begin{abstract}
Bioactive polymers with application in various industries such as pharmaceuticals, cosmetics, biomedical and food have put the emphasis on the research on microalgae. Polysaccharides isolated from micro algae have also been proved to possess several bioactive properties. Dunaliella salina is a microalga which is known for its growth in the wide range of salt concentrations. It is found to secrete complex high molecular weight biopolymers containing various components like carbohydrates, proteins, lipids etc. In the present study, extracellular polysaccharides (EPS) were isolated from Dunaliella salina to assess the bioactive properties. After 20 days of culture, the EPS was extracted by ethanol extraction and checked for the presence of carbohydrates using phenol sulphuric assay. The bioactive properties of EPS were tested on peripheral blood mononuclear cell (PBMC) and mouse macrophage cell line (RAW 264.7) based on MTT assay. Phenolsulphuric method confirmed the presence of polysaccharide in isolated EPS. It was found that EPS were able to enhance the proliferation of PBMC. In RAW cell line, stimulation was observed in the low concentration of extract whereas at high concentration, cytotoxic effect was observed. For further characterisation, the extract was tested for the presence of different compounds by thin layer chromatography (TLC) and three different peaks were observed. Hence, the present results suggest that EPS isolated from $D$. salina possess cytotoxic and immunomodulatory activities. (C) 2016 iGlobal Research and Publishing Foundation. All rights reserved.
\end{abstract}

Conference Proceedings: International Conference on Advances in Plant and Microbial Biotechnology (PMB2017); JIIT, Noida: February 02-04, 2017

Indo Global Journal of Pharmaceutical Sciences( ISSN 22491023 ; CODEN- IGJPAI; NLM ID: 101610675) indexed and abstracted in EMBASE(Elsevier), SCIRUS(Elsevier),CABI, CAB Abstracts, Chemical Abstract Services(CAS), American Chemical Society(ACS), Index Copernicus, EBSCO, DOAJ, Google Scholar and many more. For further details, visit http://iglobaljournal.com 